CHAPTER 4

\title{
SOX1 antibodies are markers of paraneoplastic Lambert-Eaton myasthenic syndrome
}

\author{
L Sabater ${ }^{1}$, MJ Titulaer ${ }^{2}$, Albert Saiz ${ }^{1}$, JJGM Verschuuren ${ }^{2}$, \\ AO Güre ${ }^{3}$, F Graus ${ }^{1}$
}

\footnotetext{
${ }^{1}$ Service of Neurology, Hospital Clinic, Universitat de Barcelona and Institut d' Investigació Biomèdica August Pi i Sunyer (IDIBAPS), Barcelona, Spain

${ }^{2}$ Dep. of Neurology, Leiden University Medical Center, Leiden, the Netherlands

${ }^{3}$ Dep. of Molecular Biology and Genetics, Bilkent University, Bilkent, Ankara, Turkey
}

The authors thank Mercè Bonastre and Eva Caballero for excellent technical assistance. 
52 | Chapter 4

Abstract

Purpose We reported that $43 \%$ of patients with Lambert-Eaton myasthenic syndrome (LEMS) and small cell lung cancer (SCLC) had an antibody, called AGNA (anti-glial nuclear antibody), defined by the immunoreaction with the nuclei of the Bergmann glia of the cerebellum. This study was undertaken to identify the antigen recognized by AGNA and to confirm the association with paraneoplastic LEMS in a larger series.

Patients and methods We probed a fetal brain cDNA library with AGNA positive sera. The presence of antibodies against the isolated antigen was detected by immunoblot of phage plaques from two positive clones. We studied 105 patients with LEMS (55 with SCLC), 50 with paraneoplastic neurological syndromes, SCLC, and $\mathrm{Hu}$ antibodies, and 50 with only SCLC.

Results Probing of the fetal brain expression library with AGNA sera resulted in the isolation of SOX1, a highly immunogenic tumour antigen in SCLC. IgG eluted from SOX1 clones produced the same cerebellar immunoreactivity as of AGNA sera. SOX1 antibodies were present in $64 \%$ of patients with LEMS and SCLC but in none of the 50 with idiopathic LEMS $(p<0.0001)$. Compared with paraneoplastic LEMS, the frequency of SOX1 antibodies was significantly lower in patients with $\mathrm{Hu}$ antibodies (32\%, $\mathrm{p}=0.002)$ and in those with only SCLC $(22 \%)$.

Conclusions SOX1 is the antigen recognized by AGNA positive sera. The detection of SOX1 antibodies in patients with LEMS predicts the presence of SCLC and may be used to follow more closely those LEMS patients with no evidence of cancer at the initial work-up. 


\section{Introduction}

The Lambert-Eaton myasthenic syndrome (LEMS) is a disorder of neuromuscular transmission mediated by voltage-gated calcium channel (VGCC) antibodies. ${ }^{1}$ Cancer, almost always, small cell lung carcinoma (SCLC), is detected in up to $50 \%$ of LEMS patients. ${ }^{1}$ Presently, there are no biological markers that predict which LEMS patients are paraneoplastic. In a previous study, we found that $43 \%$ of patients with LEMS and SCLC had an antibody, called anti-glial nuclear antibody (AGNA), defined by the immunoreaction with the nuclei of the Bergmann glia of the cerebellum. ${ }^{2}$ We observed a robust association between AGNA and SCLC. The frequency of AGNA was found higher than expected, for the simple presence of SCLC, in paraneoplastic LEMS. ${ }^{2}$ Because we observed that AGNA reactivity was widely expressed in the developing nervous system, ${ }^{2}$ we screened a fetal brain library with AGNA positive sera and identified the AGNA antigen as SOX1, a protein implicated in neural development and expressed in SCLC., ${ }^{3,4}$ We also analyzed a larger series of LEMS to confirm that antibodies against SOX1 are restricted to LEMS with SCLC.

\section{Patients and methods}

\section{Sera}

The serum of 105 LEMS patients (55 with SCLC, 50 idiopathic) was ascertained from a nationwide Dutch study (67 patients) ${ }^{5}$ and from 38 Spanish patients sent to our laboratory to detect antineuronal or VGCC antibodies. All patients presented clinical symptoms and neurophysiological studies compatible with the diagnosis of LEMS. All sera were positive for VGCC antibodies, except six LEMS patients without a tumour and one with a SCLC. The latter was tested after chemotherapy during clinical remission. Paraneoplastic LEMS required the histological confirmation of cancer. LEMS was considered idiopathic when no tumour was diagnosed after a follow-up of at least three years after the diagnosis of LEMS and serum was negative for paraneoplastic (Hu, CV2, Ri, amphiphysin, ANNA3, PCA2) antibodies usually associated with SCLC. ${ }^{1,6}$ An additional LEMS patient could not be included in any group. He was a male patient, and a lifelong smoker, who developed LEMS and cerebellar ataxia at 62 years and died at the age of 76 from stroke. Low titer of Hu antibodies were present in the serum, but cancer was never diagnosed. The presence of $\mathrm{Hu}$ antibodies would indicate a definite paraneoplastic LEMS according to recent guidelines on paraneoplastic neurological syndromes. ${ }^{6}$ 


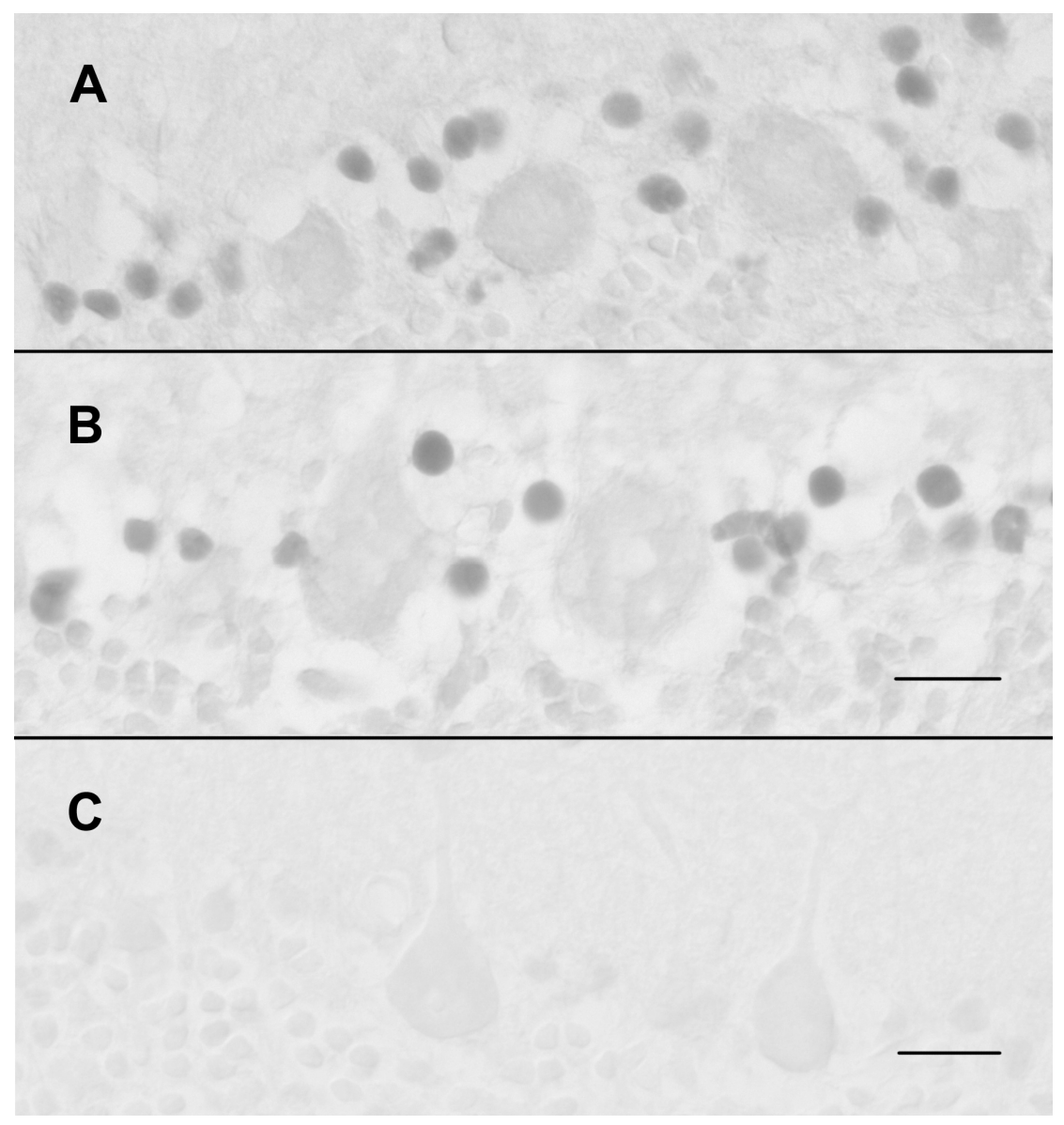

Figure 1 Frozen section of rat cerebellum incubated with an AGNA positive serum (A), and IgG from an AGNA positive serum eluted from SOX1 (B) or irrelevant (C) clones. The IgG eluted from the SOX1clone immunoreacts with the nuclei of Bergmann glia with the same pattern produced by the AGNA positive serum. Bar $=18 \mu \mathrm{m}(\mathrm{A}, \mathrm{B}) ; 24 \mu \mathrm{m}(\mathrm{C})$.

However, in the present study, we also required the histological diagnosis of SCLC to define LEMS as paraneoplastic so the patient was not included in the paraneoplastic group. As controls, we analyzed the serum of 50 patients with SCLC without neurological symptoms and 50 patients with paraneoplastic neurological syndromes, SCLC, and $\mathrm{Hu}$ antibodies (sensory neuropathy 27, cerebellar degeneration 9, limbic encephalitis 6 , encephalomyelitis 4 , sensorimotor neuropathy 3 , brainstem encephalitis 1 ). The presence of AGNA seropositivity was defined by the staining of the nuclei of Bergmann glia in standard immunohistochemistry of rat cerebellum (Figure 1). ${ }^{2}$ 


\section{Isolation of CDNA clones and detection of SOX1 antibodies}

A Uni-ZAP XR Library (Stratagene, La Jolla, CA) from human fetal brain was immunoscreened with a pool of four AGNA positive sera (each diluted 1:1000) as previously reported. ${ }^{7}$ Phage positive clones were subcloned in pBluescript using the in vivo excision phage rescue protocol (Stratagene, La Jolla, CA) and sequenced.

The presence of SOX1 antibodies in patient's sera was examined using nitrocellulose filters with mixed phage plaques $(50 \%$ of plaques from positive clones and $50 \%$ from irrelevant clones). Filters were cut into four pieces, each one incubated with different patient's sera (dilution 1:1000) and developed by an avidin biotin immunoperoxidase technique as described. ${ }^{7}$ Filters were scored by two investigators (LS and FG) blind to the clinical diagnosis. To confirm the results obtained with our SOX1 clone (clone 1) the same analysis was repeated with

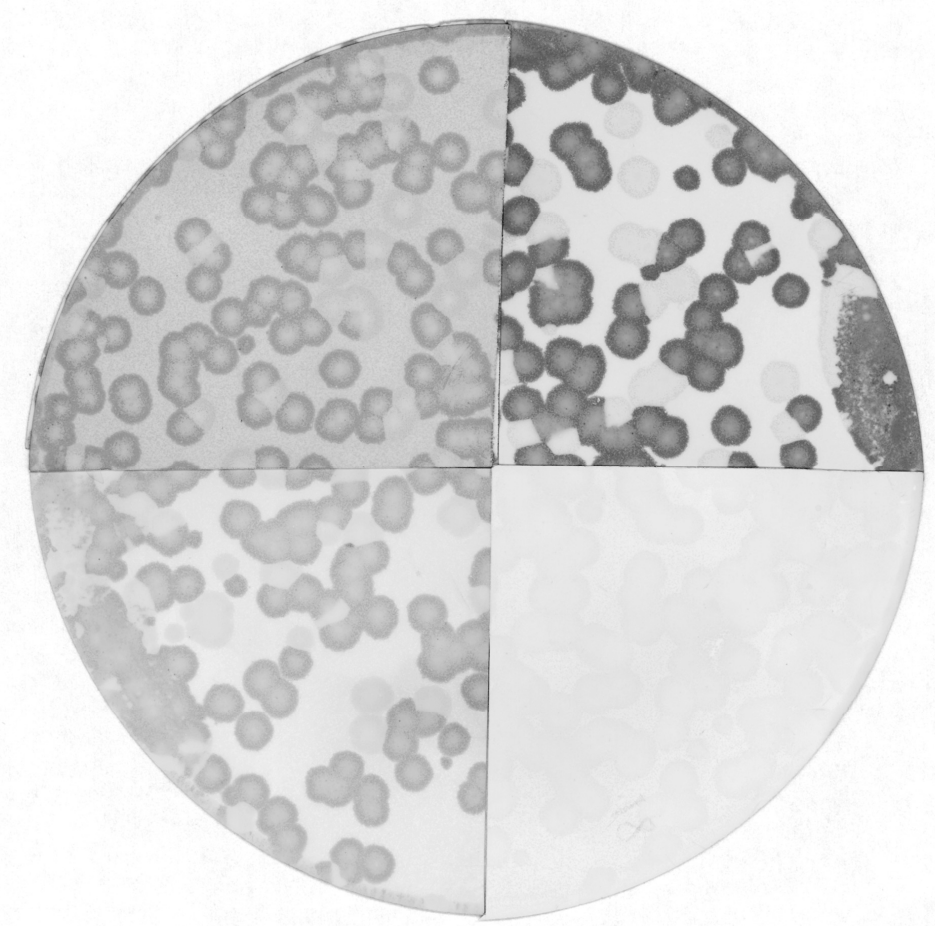

Figure 2 Detection of SOX1 antibodies with phage plaques (see methods).

Filters were cut into four pieces and used to screen patient's sera. The right lower quadrant was incubated with normal human serum, the others with three different AGNA positive sera. 
another SOX1 clone (clone 2) obtained by screening of the SCLC line $\mathrm{NCl}-\mathrm{H} 740$ with a pool of five sera from SCLC patients without paraneoplastic neurological disorders. ${ }^{4}$ Clone 2 contained the SOX1 cDNA segment 192-2261 of Y13436.1 GenBank accession number. ${ }^{4,8}$

\section{Affinity purification of antibodies}

Filters with purified phage plaques expressing SOX1 or irrelevant Escherichia Coli proteins were incubated with AGNA or control serum (dilution 1:200) for 12 hours at $4{ }^{\circ} \mathrm{C}$. After the incubation, the serum without the IgG bound to the phage plaques was collected. Filters were washed extensively and bound antibodies were eluted with sodium citrate $(\mathrm{pH} 2.5)$ and neutralized with Tris $(\mathrm{pH}$ 8.8). Purified antibodies were concentrated with a Centricon Plus-20 centrifugal filter (Millipore, Billerica, MA, USA) and the IgG measured by nephelometry. ${ }^{9}$ The eluted antibodies and the serum collected after the incubation were probed by immunohistochemistry on rat cerebellar sections to see if they reproduced the AGNA immunoreactivity.

\section{Results}

Screening of the cerebellar expression library resulted in the isolation of one clone (clone 1) identical to the human SOX1 gene (GenBank accession number NM_005986.2). Sequencing on both strands of the clone revealed a consensus sequence spanning 2952 nucleotides. The clone missed $207 \mathrm{bp}$ at the 5 ' end and contained an internal deletion of 65 bp in the segment 898-693 of NM_005986.2. This deletion was not present in the SOX1 clone (clone 2) previously described. ${ }^{4}$ (Figure S1, supplementary). The predicted translation of clone 1 had 230 aminoacids identical to SOX1 protein (GenBank accession number NP_005977.2). Although the clone lacked the first 49 aminoacids of the N-terminal end of SOX1 protein it contained the full DNA-binding domain known as HMG-box. ${ }^{10}$ The internal deletion caused an out of frame translation of 158 aminoacids of the C-terminal with no significant homologies in the databases. (Figure S2, supplementary).

Because SOX1 plaques were recognized by all the four AGNA sera used in the screening and because SOX1 is expressed in the Bergmann glia of adult cerebellum, ${ }^{11}$ we pursued further studies with this protein. To ensure the specificity of the antibody recognition, IgG was eluted from purified phage plaques expressing the SOX1 protein and incubated with AGNA positive serum. AGNA immunoreactivity was reproduced when tested by immunohistochemistry on rat 
cerebellum (Figure 1). In contrast, the serum collected after incubation with the phage plaques expressing the SOX1 protein failed to reproduce the AGNA labelling (not shown) suggesting that only the antibodies that reacted with SOX1 protein were responsible for the AGNA immunoreactivity.

Filters with plaques containing SOX1 protein of clone 1 reacted with 29 of 55 (52\%; $95 \mathrm{Cl}: 39 \%$ to $65 \%)$ paraneoplastic and 0 of 50 idiopathic LEMS ( $p<0.0001)$ (Figure 2). The same study done with the second SOX1 clone (clone 2), ${ }^{4,8}$ without the internal deletion, identified six additional sera (35/55) with SOX1 antibodies. The frequency of SOX1 antibodies was $64 \%$ (95 Cl: $48 \%$ to $75 \%$ ) in paraneoplastic and $0 \%$ in idiopathic LEMS $(p<0.0001)$. The additional LEMS patient with low titre of $\mathrm{Hu}$ antibodies but no cancer proven was positive for SOX1 antibodies.

Compared to paraneoplastic LEMS, patients with other paraneoplastic neurological syndromes, SCLC, and Hu antibodies had a lower frequency of SOX1 antibodies (16/50; 32\%; $95 \mathrm{Cl}$ : 19\% to 46\%), ( $p=0.002)$ using the clone 2 as antigen in the assay. Positive paraneoplastic cases included 10 with sensory neuropathy, three with cerebellar degeneration, two with encephalomyelitis and one with limbic encephalitis. The frequency of SOX1 antibodies in patients with isolated SCLC was $22 \%$ (95\% Cl: 13 to $35 \%$ ) (Table).

As expected from previous studies with other antibodies, ${ }^{12}$ the immunoblot assay to detect SOX1 antibodies was more sensitive than the detection of AGNA by immunohistochemistry. SOX1 antibodies were identified in all 26 paraneoplastic LEMS sera with positive AGNA immunoreactivity but also in 10 of the 29 (34\%) paraneoplastic LEMS sera that were AGNA negative or inconclusive due to the presence of concomitant antinuclear antibodies that prevented the immunohistochemical diagnosis of AGNA. ${ }^{2}$

\begin{tabular}{cccc}
\hline & $\mathrm{N}$ & $\%$ SOX1 positive & \\
\hline $\begin{array}{c}\text { LEMS } \\
\text { idiopathic }\end{array}$ & 50 & 0 & $\mathrm{p}<0.0001$ \\
$\quad$ paraneoplastic & 54 & 64 & $\mathrm{p}=0.002$ \\
Hu-positive PNS & 50 & 32 & $\mathrm{p}=0.2$ \\
SCLC alone & 50 & 22 & \\
\hline
\end{tabular}

Table PNS: Paraneoplastic neurological syndromes; SCLC: small cell lung cancer; LEMS: Lambert-Eaton myasthenic syndrome 


\section{Discussion}

This study identified SOX1 as the antigen recognized by AGNA and showed that in LEMS patients, SOX1 antibodies are very specific to predict the presence of an underlying SCLC.

SOX1 belongs to a large family of developmental transcription factor genes that share a highly conserved DNA-binding domain known as the HMG-box, defined by 79 aminoacids. ${ }^{3}$ The human SOX gene family is classified in groups from $A$ to $H$. SOX1 belongs to group B1 along with SOX2 and SOX $3 .{ }^{13}$ SOX B1 genes encode DNA-binding proteins that regulate transcription of target genes and all are strongly expressed in the early neuroectoderm and the developing nervous system. ${ }^{14}$ In the adult nervous system, SOXB1 genes are down regulated but remain active in adult neural stem cells and selected groups of differentiated neurons. ${ }^{13}$ SOX1 and SOX2 are preferentially expressed in the Bergmann glia in the adult cerebellum, ${ }^{11}$ in concordance with the immunoreactivity observed with AGNA. $^{2}$

Previous serological analysis of expression cDNA libraries (SEREX) derived from SCLC cell lines screened with sera from SCLC patients without neurological disorders, isolated SOX B1 genes confirming that the encoded proteins are expressed in SCLC and are highly immunogenic. ${ }^{4}$ Antibodies against SOX B1 proteins were present in $28 \%$ of a series of 90 patients with SCLC without neurological disorders. ${ }^{8}$ All patients with SOX2 and SOX3 antibodies were also seropositive for SOX1 and only five patients had SOX1 but not SOX2 or SOX3 antibodies. ${ }^{8}$ The common reactivity of the antibodies against all three SOX B1 proteins suggest that the immunoreactivity of SCLC patient sera is directed against epitopes of the conserved HGM box. ${ }^{4}$ Although we did not analyze the presence of SOX2 and SOX3 antibodies in our series, the previous data suggest that de detection of SOX1 antibodies is the best approach to identify the immunoreactivity of the patient's serum against SOX B1 proteins.

This study confirms for the first time that immunity against SOX1 is present in patients with paraneoplastic LEMS while being absent in non-paraneoplastic LEMS patients, suggesting that SCLC is seminal in the development of the immune response. Unlike SCLC patients with VGCC antibodies, we did not find an increased frequency of SOX1 antibodies in patients with $\mathrm{Hu}$-associated paraneoplastic neurological syndromes and SCLC. In fact, the frequency of SOX1 antibodies (32\%) in patients with Hu-associated paraneoplastic neurological syndrome and SCLC was not significantly different from that found in the patients 
with isolated SCLC (22\%) in line with the previously reported frequency (28\%) using the same clone (clone 2 ) as antigen. ${ }^{8}$ These observations could indicate that the individual variables that are determinant to mount an immune response against the VGCC of the SCLC are also relevant to raise a SOX1-mediated tumour immunity.

In a previous study, we found AGNA in two patients with limbic encephalitis, SCLC and voltage-gated potassium channel (VGKC) antibodies. ${ }^{15}$ No AGNA reactivity was found in patients with limbic encephalitis, SCLC and absence of VGKC antibodies or those with non-paraneoplastic limbic encephalitis and high titers of VGKC antibodies. ${ }^{16,17}$ As in the current work, we have confirmed that AGNA immunoreactivity in limbic encephalitis patients is caused by SOX1 antibodies (unpublished). Taken together, these data suggest that, in the setting of a SCLC, there is a close association between the presence of SOX1 antibodies and antibodies against neuronal membrane channels that will require further studies.

When neurologists evaluate a patient with LEMS, there are no robust clues that predict the presence of an underlying cancer. Paraneoplastic neuronal or muscle antibodies are reported in up to $37 \%$ of patients with paraneoplastic LEMS but their predictive value remains unclear. ${ }^{18}$ In a previous study, we found that the absence of HLA-B8 and a positive smoking history to be highly sensitive (88\% and $96 \%)$ for the presence of SCLC but both variables had a relative low specificity $(<70 \%){ }^{5,19}$ We demonstrate that the detection of SOX1 antibodies was restricted to LEMS patients with histologically proven SCLC, with one exception. The clinical profile of this particular patient suggests that the tumour could had been controlled by the immune response as reported previously. ${ }^{20}$ In conclusion, the detection of SOX1 antibodies is a simple test that may be used to define a better model to predict the presence of SCLC in LEMS patients.

\section{References}

1. O'Neill J.H., Murray N.M.F., Newsom-Davis J.: The Lambert-Eaton myasthenic syndrome - A Review of 50 Cases. Brain 111:577-596, 1988

2. Graus F., Vincent A., Pozo-Rosich P. et al: Anti-glial nuclear antibody: marker of lung cancer-related paraneoplastic neurological syndromes. $\mathrm{J}$ Neuroimmunol 165:166-171, 2005 
3. Wegner M., Stolt C.C.: From stem cells to neurons and glia: a Soxist's view of neural development. Trends Neurosci. 28:583-588, 2005

4. Güre, A.O., Stockert, E., Scanlan, M.J., et al: Serological identification of embryonic neural proteins as highly immunogenic tumor antigens in small cell lung cancer. Proc Natl Acad Sci USA 97:4198-4203, 2000

5. Wirtz P.W., Willcox N., van der Slik A.R., et al: HLA and smoking in prediction and prognosis of small cell lung cancer in autoimmune LambertEaton myasthenic syndrome. J Neuroimmunol 159:230-237, 2005

6. Graus F., Delattre J.Y., Antoine J.C., et al : Recommended diagnostic criteria for paraneoplastic neurological syndromes. J Neurol Neurosurg Psychiatry 75:1135-1140, 2004

7. Bataller, L., Wade, D.F., Graus, F., Rosenfeld, M.R., Dalmau, J.: The MAZ protein is an autoantigen of Hodgkin's disease and paraneoplastic cerebellar dysfunction. Ann Neurol 53:123-127, 2003

8. Vural B., Chen L., Saip P., et al : Frequency of SOX group B (SOX 1, 2, 3) and ZIC2 antibodies in Turkish patients with small cell lung carcinoma and their correlation with clinical parameters. Cancer 103:2575-2583, 2005

9. Sabater L., Gómez-Choco M., Saiz A., Graus F.: BR serine/threonine kinase 2: A new autoantigen in paraneoplastic limbic encephalitis. J Neuroimmunol 170:186-190, 2005

10. Malas S., Duthie S.M., Mohri F., et al: Cloning and mapping of the human SOX1: a highly conserved gene expressed in the developing brain. Mamm Genome 8:866-868, 1987.

11. Sottile V., Li M., Scotting P.J.: Stem cell marker expression in the Bergmann glia population of the adult mouse brain. Brain Res 1099:8-17, 2006

12. Graus F., Dalmau R., Reñé R., et al: Anti-Hu antibodies in patients with small-cell lung cancer: Association with complete response to therapy and improved survival. J Clin Oncol 15: 2866-2872, 1997

13. Schepers G.E., Teasdale R.D., Koopman P.: Twenty pairs of Sox: Extent, homology, and nomenclature of the mouse and human Sox transcriptor factor gene families. Dev Cell 3:167-170, 2002

14. Pevny L., Placzek M. SOX genes and neural progenitor identity. Curr Opin Neurobiol 15:7-13, 2005 
15. Zuliani L., Saiz A., Tavolato B., et al: Paraneoplastic limbic encephalitis associated with potassium channel antibodies: value of anti-glial nuclear antibodies (AGNA) to identify the tumour. J Neurol Neurosurg Psychiatry 78:204-205, 2007

16. Pozo-Rosich P., Clover L., Saiz A., et al: Voltage-Gated Potassium Channel antibodies in limbic encephalitis. Ann Neurol 54:530-533, 2003

17. Vincent A., Buckley C., Schott J.M., et al: Potassium channel antibodyassociated encephalopathy: a potentially immunotherapy-responsive form of limbic encephalitis. Brain 127:701-712, 2004

18. Schmalstieg W., Lachance D., Lennon V.: Prognosis and autoimmunity in Lambert-Eaton syndrome. Neurology 68 (Suppl 1):A107, 2007

19. Wirtz P.W., Roep B.O., Schreuder G.M.Th., et al : HLA class I and II in Lambert-Eaton myasthenic syndrome without associated tumor. Human Immunology 62:809-813, 2001

20. Darnell R.B., DeAngelis L.M.: Regression of small-cell lung carcinoma in patients with paraneoplastic neuronal antibodies. Lancet 341: 21-22, 1993 


\section{Supplementary figures}

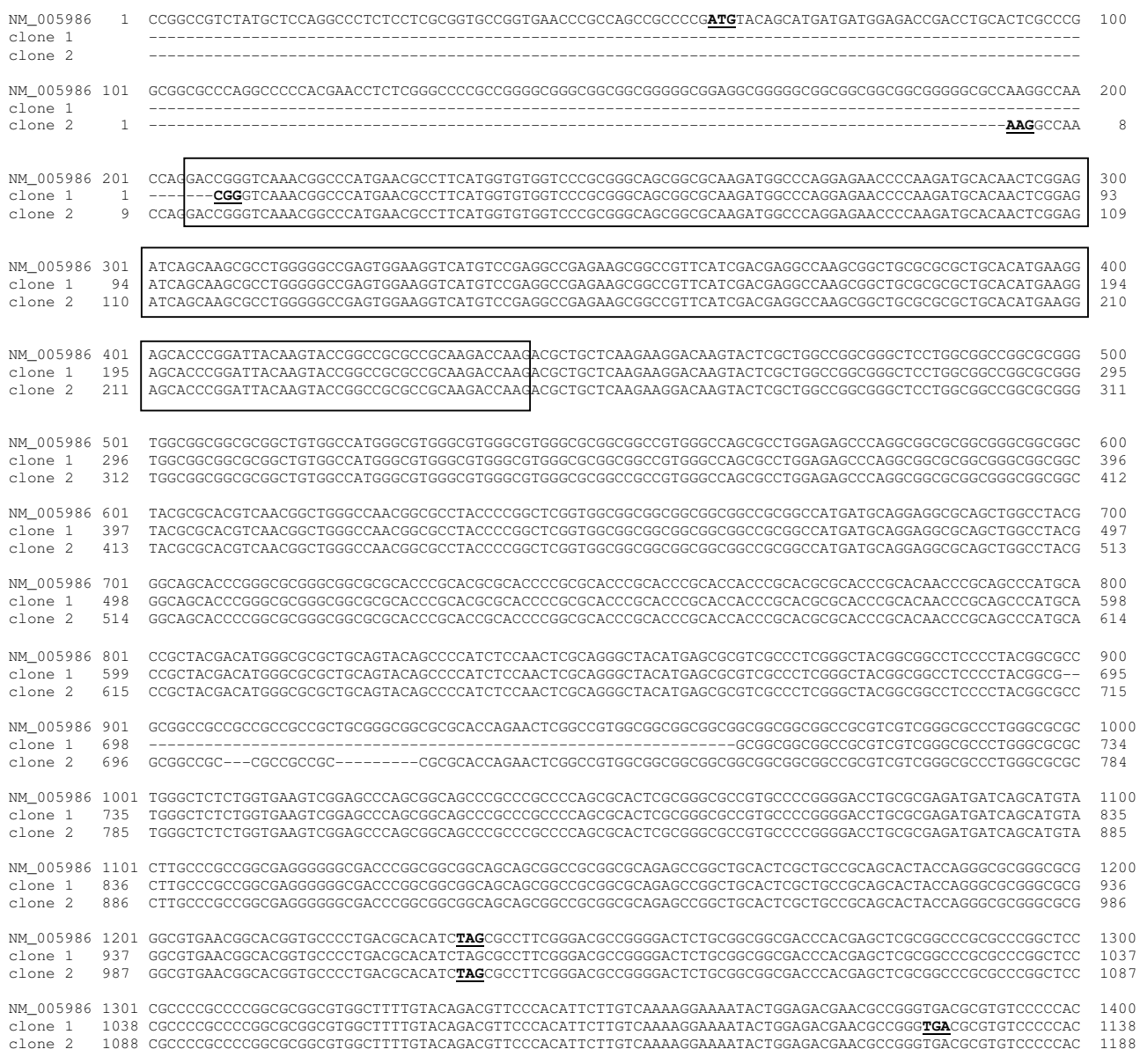

Figure S1 Comparison of the two clone sequences (see text) with the canonical NCBI entry NM_005896, showing only the coding region. The 3 ' untranslated region was identical in the three sequences. The HMG sequence is depicted in a box. The start and the stop codons of each sequence are in bold and underlined. Deletions at the $5^{\prime}$ end and in the internal region are indicated with a (-). 
SOX1 in LEMS | 63

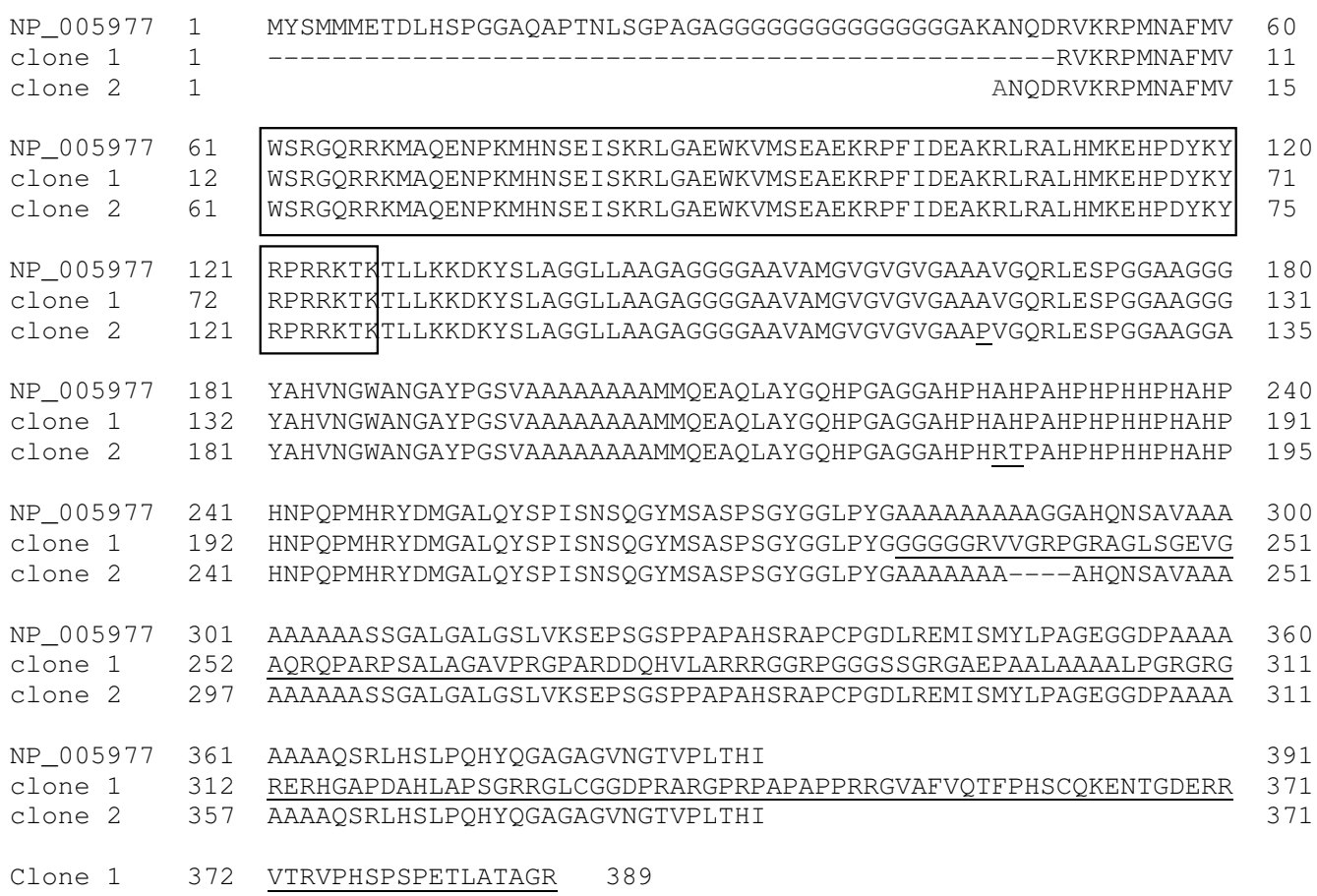

Figure S2 Homologies of the predicted aminoacidic sequence of the clones with the human SOX1 NCBI entry. Differences are underlined. The HMG motif is depicted in a box. 
